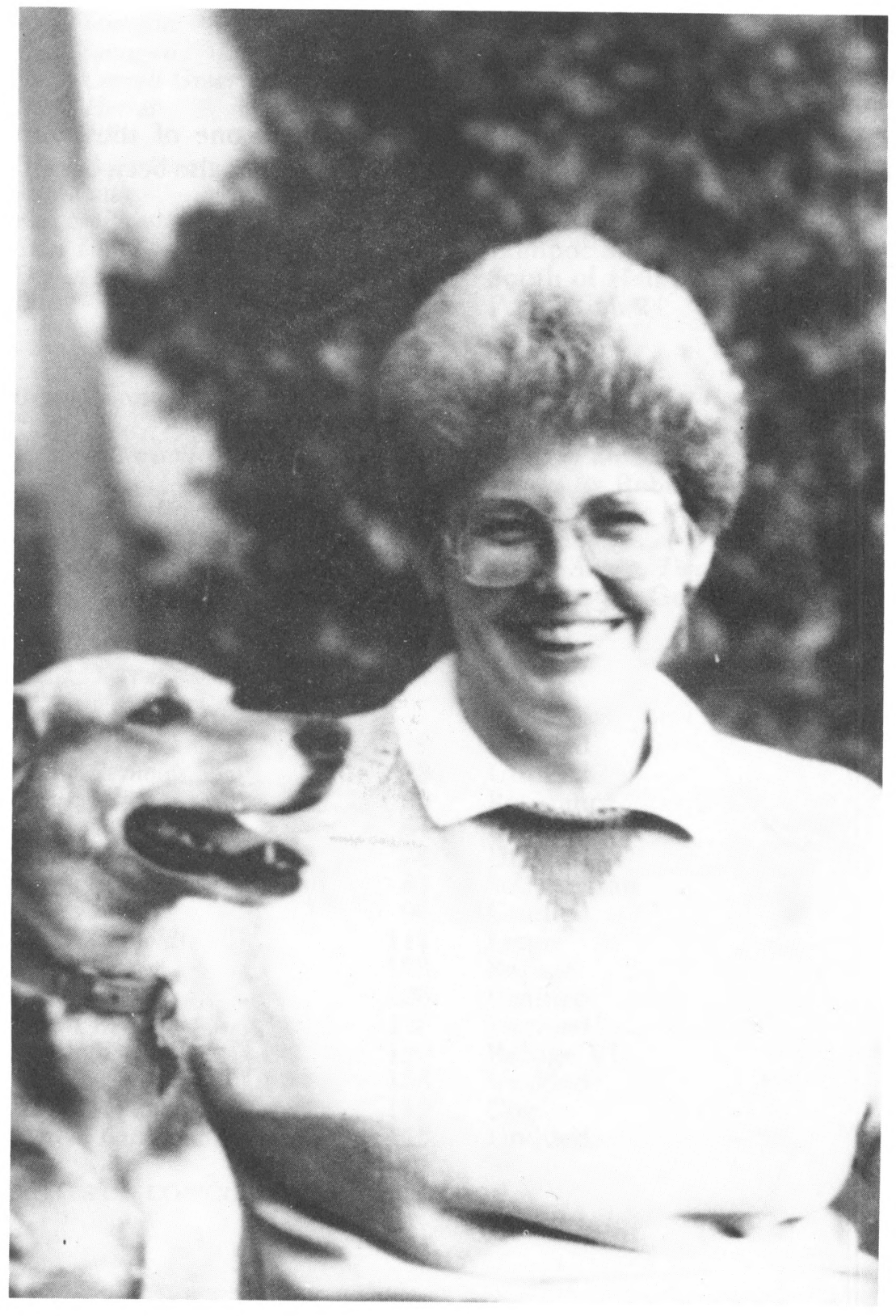




\section{INTERVIEW WITH N. KATHERINE HAYLES}

Suzanne Araas-Vesely

KATHERINE HAYLES HAS unusual credentials for a literary scholar and critic. She has a master's degree in Chemistry from the California Institute of Technology (1969). She has taught literature at Dartmouth College, the California Institute of Technology, and the University of Missouri-Rolla. In 1985 she came to the University of Iowa, where she has been awarded a faculty scholarship, 1986-89. She has made presentations at numerous conferences, has written articles on subjects ranging from Renaissance drama to modern fiction, and is the author of one book, The Cosmic Web: Scientific Field Models and Literary Strategies in the Twentieth Century, and two forthcoming studies, including Chaos Bound: Orderly Disorder in Contemporary Literature and Science, due out the first of next year. In 1991 she will take over the presidency of the Society for Literature and Science.

Kate, could you acquaint our readers with your field and why it may be important to the study of literature and language?

The first thing I notice about this question is that it's phrased in the singular. The Cosmic Web was not so much about a single field, as about a field as an interactive relationship between subject and object. There are, for example, any number of physical fields one could talk about - the dynamic field, quantum mechanical field, and so forthbut the field as a concept has as its core the denial of the strict separation of subject and object. It's in this sense that it's important to range literature and language alongside field theories, because literature and language are central to the relationship between subject and object.

In your book The Cosmic Web you mention the 1979 article by Evelyn Keller, "The Cognitive Repression in Contemporary Physics," which suggests that sexist assumptions on the part of mostly male contemporary physicists, 
especially as they are linked to the belief in the world occupied by subjects and objects, is a barrier to creating a completely successful unified field model. Has there been any type of unified field theory developed since you wrote your book that has overcome the subject-object dichotomy? Is it still, as you were saying, just a set of fields rather than a single field?

There has been progress on various technical and scientific fronts. For example, super-string theory is now calling for a radical revision of the components of reality conceptualized by classical physics. Instead of four dimensions, super-string theory envisions anywhere from ten to twenty-four. In the case of ten dimensions, six of them are so tightly curled that only four can be seen. Super-string theory thus expands the vision of an interactive relationship implicit in early field theories. But when you speak about overcoming subject-object dichotomy, you're obviously talking about more than scientific paradigms or theories. You're talking about people's relationships with other people, to the world around them, and so forth. And this is such a complex question, reinforced by culture, language, institutions, that I don't think there's any possibility that a scientific theory of any kind could overcome them. What one could expect is an incremental difference in how we relate to the world around us, and in how we conceptualize that relation. But I don't think it's something that's going to achieve any one final form.

If the subject-object dichotomy is still a conceptual problem for scientists, what role does the literature need to play in fulfilling the end of science, maybe using "end" in a double sense?

That's a very complex question. It's easy to point to literary works that have been influenced by science, but it's very difficult to point to scientific works that have been overtly influenced by literature. To the extent that influence goes from literature to science, I think it does so in the ways that Gillian Beard describes, through narrative structures that are embedded within scientific discourse. For example, any scientific model that traces behavior through time could potentially constitute a narrative. Similarly, syntactic patterns, semantic usages, and metaphoric linkages also enter into scientific writing. So there is this indirect kind of influence from literature. To the extent that twentieth-century literature, especially, has been responsive to imagining different ways of being, it does in fact play a role in fulfilling the end of science.

I think it's an exciting possibility. And I'm also wondering how many 
contemporary physicists read Pynchon's work? Of course, that guiding role of literature might be a matrix type of role rather than, as you said, depending on direct influence. Do you think that the model of what you call a "cultural matrix" would require Whitehead's "climate of opinion" to be in place before, say, Einstein's and Saussure's independent efforts could both suggest a relation of field models? Or can an individual apprehend a physical-cultural matrix?

Individual creative acts spring from the interaction between an individual's sensibility and the cultural climate in which that individual works. When an individual vision is very idiosyncratic or very strongly motivated internally, it's quite possible that a vision can emerge that works at cross-purposes with the prevailing cultural climate. A good example, I think, would be William Blake.

Yes. He is still interacting with the cultural matrix around him, but there seems to be a lot that is unusual; the alternative representations of what passes for truth in the text by the illustrations force the reader to keep questioning her or his assumptions. Blake seems almost modern in that respect; you don't dare ignore anything.

Yes. Strong evidence for the effect of culture on all kinds of creative acts is demonstrated by the fact that important scientific discoveries are often made simultaneously, or nearly so, in widely separated sites. And this happens between science and other areas as well. Art, literature, theology, philosophy, very often manifest important correlations with one another. And the most compelling explanation for this phenomenon is cultural.

To change the subject, I'm wondering how much the study of earlier literature such as eighteenth-century British, for example, really even very early modern, can profit from a "field" approach that is only spoken of and thought of in our cultural matrix. How unified can this field of literature be with regard to the metaphor of the unified field?

I think that's a really important question, and it's one that I come back to again and again, without arriving at any easy resolution to it. At the moment, what most satisfies me is to say that similar concepts can and do occur to thinkers in any period. But that if there are not enough other people thinking along the same lines, if the idea is not strongly reinforced in the culture, then it is not likely to enter into the cultural mainstream and to become part of its tradition. It exists, it may even endure because of individual genius, but it will not have the same kind of effect as when a cultural moment is right for crystallization to occur. 
It almost sounds like you're talking about layers of creation or reality or time, you know, like rings on a tree, or better yet, exchanges between neurotransmitters in a living organism.

There's definitely a feedback loop, I think, between the individual creative act and the cultural context. The culture no doubt influences the individual creative act; and individual creative acts also enter into and influence the culture as a whole.

This feeds into my interest in earlier centuries because it seems to justify keeping some sense of the canon from an earlier age, not necessarily insisting on what ingredients should be in there but, more importantly, what insights are related to ours or even ahead of ours.

Exactly. We can see threads of rich anticipation. I don't deny that those are really there, but I also don't deny that it's our particular cultural movement which focuses our attention upon them. I see literary works as variegated tapestries out of which many patterns and threads could be followed. What a particular time chooses to focus on reflects a particular cultural moment. The full tapestry is the result of complex interactions between individual sensibilities, the traditions in which works evolve, and the cultural context that energizes some concerns and not others.

In your section on science, you mentioned that there are inevitable indeterminicies, even in mathematics, such that the concept that an absolutely pure mathematics in which everything is proven is no more tenable than the positivist's attempt to create an unambiguous scientific language. Do you think that a change in the way scientists write would be good or feasible at this time? And if so, what kind of writing would best be able to articulate a unified field? I sometimes have thought scientists ought to be required to present their theories as plays or musicals and perform them in conferences.

[Laughs.] I love the idea of performing physical theory as a musical or play; that's a wonderful idea that people in the biology department here are actually working upon, setting chaotic data to music so that the ear, with its sophisticated processing, can discern patterns too subtle or complex for mathematical analysis. Whether scientists should write differently very much depends upon the audience and context. There's no question that the conventions of scientific writing embody presuppositions that most people working in the genre are not aware of. Moreover, these presuppositions sometimes work 
against the conceptual content of the articles. The question of what conventions and modes of writing best fit specific purposes within disciplines seems to me complex. I don't know that someone from another discipline ought to impose his or her ideas about what "good" writing is, at least not without looking carefully at the disciplinary context for the discourse. Having said that, I would add that scientific writing as a mode of discourse strongly reinforces the idea of the subject-object split. If we wanted to create a mode of discourse that would attempt to overcome the subject-object dichotomy, it would be necessary to think deeply about how the presupposition of objectivity is embodied within scientific writing.

You have just defined the problem with science, if I understand you correctly, as a problem with how language is perceived: because language is a tool of observation within the field, like any other tool of science it purports to describe what it is immersed in and ends up referring back to itself. Here science begins to look more like literature, at least like contemporary literature. In The Cosmic Web you remind us of the isomorphism of the history of scientific and literary questions that Derrida spoke of in Of Grammatology, but for you it is most rooted in Descartes where Derrida finds the root primarily in Kant. It seems as if there can be more than one cultural matrix in an age, more than one cosmic web.

You're touching here on an aspect of my first book that I try to go beyond. In The Cosmic Web I hypothesized a cultural matrix without really looking at what it meant or how it works. In my second book, Chaos Bound, I've tried to go more deeply into the questions and, at the same time, to pose a more complex model for how interactions between disciplinary inquiries and cultural institutions take place. You're quite right to suggest that it's too simple to think of one cultural matrix in an age, because clearly culture is highly fissured and stratified, not by any means homogeneous or monolithic.

In Cosmic Web there was a phrase, "speaking from within the field." To my way of thinking, that's a most important concept, because it explains the shift in writing that has brought the self-referential nature of language into focus. But if all language is part of a field, oughtn't any writing be able to speak this selfreferentiality, as Barthes demonstrates in the case of Balzac's "Sarrasine?"

That goes back to what I was saying earlier about literary works being rich tapestries in which various epochs choose to follow some threads over others. For example, self-referentiality was not invented in the twentieth century. Midsummer Night's Dream is a case in point. What's 
different about reflexive writing in the twentieth century is its strong reinforcement by other strands within culture, so it comes to have a prominence that earlier reflexive writing did not.

This "speaking from within the field," just to press a little bit further... Barthes uses a similar expression when he says "the text speaks itself" When you were speaking at the University of Iowa conference on Creativity and Research on March 7th, 1989, you spoke of your best creative moments as a collaboration with a "submerged voice" which tells you "what you didn't know you knew." Is this "speaking from within the field?" It also interests me that you described this experience in terms of voice, when Derrida tries to privilege writing over voice as the basic means of expression. Are you talking about the same thing as Derrida's "trace?" How do you achieve such a powerful flow of creative ideas? Do you have any suggestions, to get practical for a minute?

This conversation within ourselves takes place through speaking, as well as through writing. One way to open that creative flow is to write in an interactive way, to let the writing serve as a medium between what you know you know and what you don't know you know. Is this "speaking frow within the field?" That's an interesting question. I've been teaching a course this term with David Klemm and Bill Klink on reflexivity. I've had occasion to reconsider these questions, and also put them in a context of theological and scientific texts. Martin Buber's I and Thou is a wonderful example of what it would mean to be in communication not only with the "Thou-ness" of another person, but also with the "Thou-ness" within one's self. We were talking in class about the contrast between Buber's approach and Freud's. Whereas Freud's strategy was to objectify the parts of the self with which we are not in intimate and continuous communication, Buber's approach suggests that it's only when we see ourselves as a unified self permeated by "Thou-ness" that we can achieve this internal communication.

Is it possible to have such a unified self when, for example, you have somebody like Pynchon pointing out in Gravity's Rainbow how many different selves can exist in one individual?

I don't think it could be achieved as a continuous state, but I think there are moments in which one does feel this. And of course the moment one is aware of the feeling, one has already lost it. From that point of view, the act of speech fractures the field. One does speak always from within the field, but the act of speech also fractures the field that contains it. 
And yet it seems to be groping for a way to make it more permanent.

That's right. That's the paradox. Becoming aware of this internal communication is a way to try to make it more permanent, but by the very act of possessing it, one also loses it. It's a way to try to make it more permanent, but by the very act of making it more permanent, it also in an important sense loses it.

If, as you are suggesting, a field theory approach to literature goes beyond the assumptions of post-structuralism, as you indicated in your remarks on Derrida, are there any more important ways it does this, other than what you've touched on?

One of the elements that I would like to put into pictures $I$ construct is the interaction between culture and text. For me this is an important way in which I want to go beyond. Actually, I'm uneasy with "going beyond"- "adding to" or "departing from," maybe-other works in the field, including my own earlier efforts.

And now, as a result, your new book Chaos Bound is a dealing with chaos theory as a richer way science has of thinking about order, as always containing asymmetries, never exact. You were talking about not "going beyond" so much as Chaos Bound's "emerging out of" The Cosmic Web.

I see the two studies as very much related. The deeper indications of field concept became apparent in a number of different areas when it was realized that a complete articulation of a formal system was not possible. Had these attempts to articulate completely formal systems succeeded, the result would have been an all-encompassing order. But since they were not possible, the pendulum began to swing the other way, and people became interested in disorder and chaos, in that which escapes resolution and cannot be predicted. It's too early to say where that pendulum swing is going to stop; but the idea that such a swing is happening is central to Chaos Bound. The field concept had its greatest impact in the first half of the century; in the second half of the century, paradigms of disorder are coming to the fore. The Cosmic $W e b$ and Chaos Bound are complementary in this sense. I like to think that together, each signifies more than it could by itself. 